\title{
Ultrasound-Energy Consumption During Phases of Phacoemulsification of Nuclear Cataracts Using Femtosecond Laser: A Comparative Study
}

This article was published in the following Dove Press journal: Clinical Ophthalmology

\author{
Ahmed H Assaf ${ }^{1,2}$ \\ Bassem F Aziz (iD) ${ }^{1,2}$ \\ 'Ophthalmology Department, Faculty of \\ Medicine, Ain Shams University, Cairo, \\ Egypt; ${ }^{2}$ Al Watany Eye Hospital, Cairo, \\ Egypt
}

Purpose: To compare ultrasound (US) energy utilized in different phases of nuclear cataract removal in femtosecond laser-assisted cataract surgery (FLACS) with conventional phacoemulsification surgery (CPS) in relation to different nuclear densities.

Methods: A prospective nonrandomized comparative study was conducted at Ain Shams University and Al Watany Eye Hospital, Cairo, Egypt on 250 eyes with senile nuclear cataracts (NCs) of different nuclear densities (1-6). Eyes were divided into two groups FLACS and CPS - and each group was subdivided according to cataract density into subgroups A (NC 1-2), B (NC 3-4) and C (NC 5-6). sextant-softened fragmentation patterns were assessed in the FLACS group and the quick-chop technique used in the CPS group. US energy required for nucleus cracking (EFX split) and for quadrant removal (EFX quadrant) was recorded.

Results: Total eyes included in the FLACS and CPS groups were 117 and 133, respectively. No significant differences between the groups for EFX quadrant and EFX split groups were observed ( $P=0.18$ and $P=0.49$, respectively). For subgroup A, no significant difference was found between FLACS and CPS on EFX split $(P=0.08)$ and EFX quadrant $(P=0.49)$. For subgroup B, significantly lower values of EFX split $(P=0.0001)$ and EFX quadrant $(P<0.0001)$ were obtained with FLACS than CPS. For subgroup $\mathrm{C}$, no significant difference was found for EFX split $(P=0.86)$; however, EFX quadrant was significantly lower in the FLACS group $(P=0.05)$.

Conclusion: FLACS lowers US energy utilized during quadrant removal at different nuclear densities, with highest significance in medium-density nuclear cataracts. Nucleus cracking by femtosecond laser is less effective in very hard cataracts. However, femtosecond-laser softening of hard nuclei is capable of of US-energy reduction during quadrant removal.

Keywords: phacoemulsification, femtosecond laser, FLACS, cataract surgery

\section{Introduction}

Conventional phacoemulsification surgery (CPS) is the standard procedure for cataract extraction nowadays with excellent outcomes. ${ }^{1,2}$ However, femtosecond laser-assisted cataract surgery (FLACS) represents a potential paradigm shift in cataract surgery, yet with considerable controversy. ${ }^{3}$ The femtosecond laser (FL) delivers ultrashort $\left(10^{-15}\right.$ seconds) pulses of energy at near-infrared wavelengths that can be precisely focused at various depths in the anterior segment of the eye. ${ }^{4}$

Owing to its photodisruptive effect and subsequent precise tissue cutting, the FL has been promoted to improve many of the critical steps of CPS. ${ }^{5,6}$ These steps
Correspondence: Ahmed H Assaf Ophthalmology Department, Ain Shams University, Cairo, Egypt. Assaf Clinic, 52 Abu El Atahya Street, Nasr City, Cairo, Egypt

Tel +20 $22270-6761$

Email assaf.ahmed@gmail.com 
include astigmatic limbus-relaxing incisions, corneal wound construction, anterior capsulotomy/laser-incised capsulorhexis, and lens pretreatment using liquefaction or fragmentation patterns to segment the nucleus and soften harder cataracts. $^{7}$ It has been postulated that FL usage in these domains reduces the magnitude of ultrasound (US) energy delivery and reduces the potential of trauma to surrounding structures during lens extraction, ${ }^{8,9}$ thus leading to faster and safer cataract surgery with better visual outcomes. ${ }^{10}$

Regarding cataract density, a number of large studies and meta-analyses have found comparable differences regarding the safety and efficacy of both FLACS and CPS when treating medium cataracts. ${ }^{11}$ However, lens fragmentation using FL has an upper limit of capability in Lens Opacities Classification System grade 4 cataracts, and thus brunescent cataracts may require CPS or even extracapsular-cataract surgery. ${ }^{12}$ This cataract type has been excluded from many of the published cohorts and deserves further investigation. ${ }^{11,13}$ The aim of our study was to compare USenergy consumption between CPS and FLACS during both the nuclear splitting stage (EFX split) and quadrant-removal stage (EFX quadrant) at different nuclear densities.

\section{Methods}

This prospective nonrandomized comparative study was conducted at Ain Shams University Hospital and Al Watany Eye Hospital, Cairo, Egypt, between March 2018 and March 2019. Eyes with visually significant senile nuclear cataracts (NCs) in which corrected distance visual acuity was $<0.5$ (decimal) were included. All participants were adequately informed about the advantages, disadvantages, and cost of both FLACS and CPS by medical staff. Both procedures were presented as valid options to all patients, and the final decision was based on patient preference after signing a consent form.

Both CPS and FLACS groups were further subdivided into three subgroups according to nuclear density and color based on the Lens Opacities Classification System III. Also, nuclear grading was done using 3-D lens densitometry with Pentacam nucleus staging (Figure 1), included with Pentacam software (Oculus, Wetzlar, Germany). Nucleus staging results were a classification of each case as one of six stages (0-5). Additionally, a clear 3-D reconstruction of lenses with visible areas of opacification is offered.

In our study, $\mathrm{NC}$ density and color were determined on a scale of 1-6 for all eyes. Subgroup A included eyes with NC1-2, subgroup B; NC3-4, and subgroup C; NC5-6. Eyes with corneal opacities, zonular weakness, subluxated lenses, maximum pupillary dilation $<5.5 \mathrm{~mm}$, iris abnormalities, history of glaucoma/uveitis, intraoperative complications, or any other comorbidity that would have complicated cataract surgery were excluded. This study adhered to the tenets of the Declaration of Helsinki and was approved by the local ethics committee of Al Watany Eye Hospital, Cairo, Egypt.

\section{Surgical Techniques}

All surgeries in the FLACS and CPS groups were performed under topical anesthesia by the same experienced high-volume surgeon (AA).

\section{FLACS Group}

FL treatment was done using the Catalys precision system (Johnson \& Johnson Vision) prior to US phacoemulsification and intraocular lens (IOL) implantation. Initially, the suction cone of the Liquid Optics Interface (was positioned on the limbus and the vacuum was activated. Once suction and ocular fixation had been confirmed, the suction cone was filled with balanced saline solution and then the eye was docked to the system. The dimensions of the anterior chamber and crystalline lens were then measured by 3-D spectraldomain optical coherence tomography, which is incorporated in the Catalys laser platform. Identified ocular structures in the anterior segment of the eye were overlaid by preprogrammed treatment zones and displayed to the surgeon for verification and/or redesigning the treatment plan. Finally, the selected treatment was initiated.

The FL was used for capsulotomy, lens fragmentation, and astigmatic keratotomies to address corneal astigmatism in some eyes. The FL made neither primary nor secondary corneal incisions. For lens prefragmentation, we used sextants with softening fragmentation function (Figure 2). Grid spacing was set at $350 \mu \mathrm{m}$, segmentation-softening spacing at $200 \mu \mathrm{m}$, and fragmentation diameter was set to the maximum. Segmentation repetition was set at 2-3 in soft nuclei of subgroup A, 3-4 in harder nuclei of subgroup $B$, and 5-6 in brunescent nuclei of subgroup C. Other laser settings were kept at default values: horizontal spot spacing $10 \mu \mathrm{m}$, vertical spot spacing $40 \mu \mathrm{m}$, anterior pulse energy $10 \mu \mathrm{J}$, posterior pulse energy $40 \mu \mathrm{J}$, anterior-capsule safety margin $500 \mu \mathrm{m}$, and posterior-capsule safety margin $500 \mu \mathrm{m}$.

Phacoemulsification for the FLACS and CPS Groups The same phacoemulsification machine (Signature WhiteStar FX, Johnson \& Johnson Vision) was used for all eyes. 


\section{OCULUS - PENTACAM Scheimpflug Images}

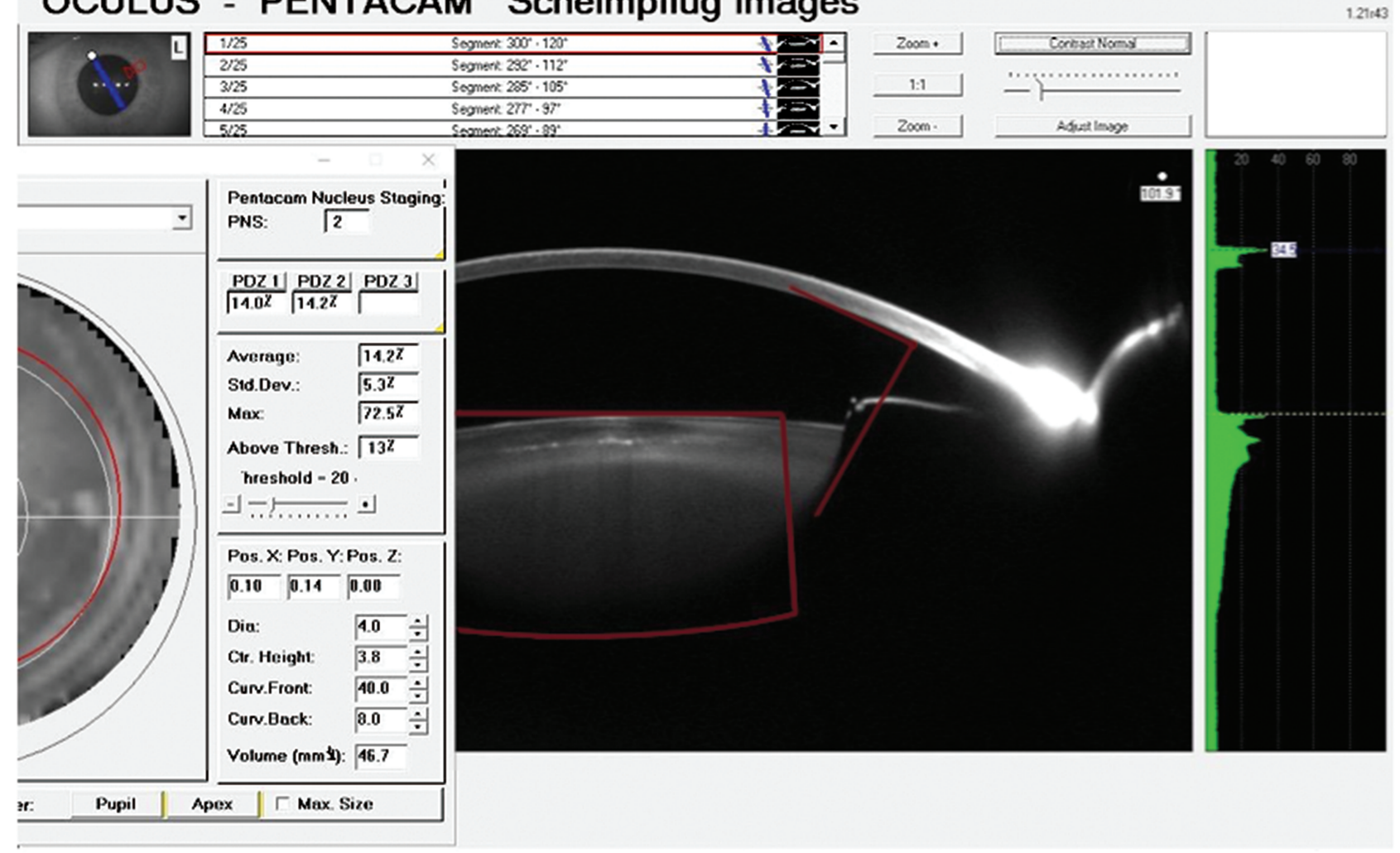

|

Figure I Pentacam nucleus staging (PNS).

Phacoemulsification settings used in both groups were US power $10 \%-40 \%$, Ellips FX transverse US phacoemulsification handpiece, WhiteStar variable duty cycle 1 , aspiration rate $28-32 \mathrm{~cm}^{3} / \mathrm{min}$, vacuum $350 \mathrm{mmHg}$ max, CASE mode, and CASE vacuum $280 \mathrm{mmHg}$. Main incisions and secondary incisions were performed using $2.2 \mathrm{~mm}$ and $1.2 \mathrm{~mm}$ keratomes respectively. An ocular viscosurgical device (sodium hyaluronate $1.3 \%$ [Healon GV], Johnson \& Johnson Vision) was injected into the anterior chamber. Manual capsulorhexis of 5-5.5 mm diameter was performed using capsulorhexis forceps in the CPS group. In the FLACS group, the capsulotomy button was removed using capsulorhexis forceps. Hydrodissection was performed in the usual way in both groups. However, it was gentle and with less fluid in the FLACS group to release trapped gases from underneath the nucleus into the anterior chamber. This was followed by the introduction of a $21 \mathrm{G}$ laminar-flow phacoemulsification tip through the main incision. In both groups, minimal US energy coupled with high vacuum $(350 \mathrm{mmHg})$ was used to impale the phacoemulsification tip within the central part of the nucleus for fixation. In the CPS group, a quick-chop technique was implemented on the nucleus using a Neuhann vertical chopper (Geuder). In the FLACS group, the same technique was used, and mechanical cracking of the nucleus into sextants was performed along preformed laser cuts.

After phacoemulsification, a foldable hydrophobic IOL was inserted into the capsular bag using the corresponding specific unfolder for each IOL. The same postoperative treatment was administered to all patients: prednisolone acetate $1 \%$ eyedrops (Pred Forte, Allergan) five times daily for 2 weeks, then gradual withdrawal over 2 weeks and topical moxifloxacin hydrochloride ophthalmic solution $0.5 \%$ (Vigamox, Alcon) five times daily for 10 days.

The EFX, which is a unitless value, denotes US\% and US time utilized during phacoemulsification and corresponds roughly to the effective phacoemulsification time (EPT) with a specific coefficient for transverse movement expressed in seconds. EFX was the primary study parameter and recorded for all eyes at two phases: after nucleus disassembly (first phase, EFX split) and after the end of the surgery (second phase, EFX total). The US required for the quadrant-removal phase (EFX quadrant) 


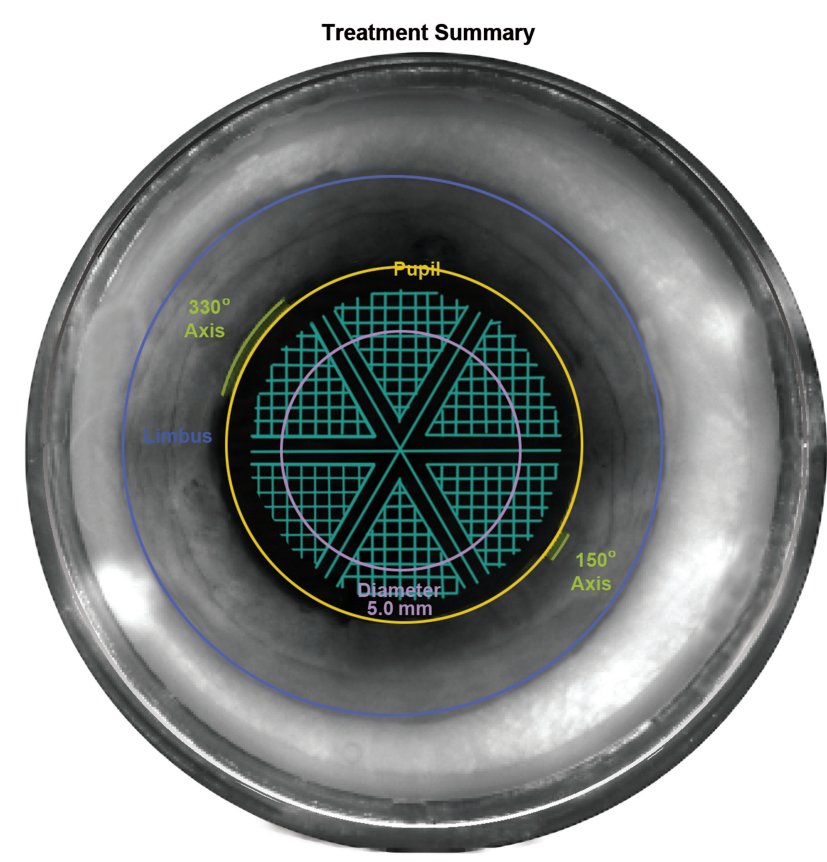

Figure 2 Lens fragmentation used in all cases in the FLACS group: sextants with lens softening, grid spacing $350 \mu \mathrm{m}$, seg-soft spacing $200 \mu \mathrm{m}$.

was calculated by subtraction of EFX split from EFX total. Average US\% and US time in seconds were also documented and compared.

\section{Statistical Analysis}

Data analysis was performed using MedCalc version 18.9.1 (MedCalc Software, Ostend, Belgium). Normality of data samples was evaluated by means of the D'AgostinoPearson test. The Mann-Whitney test, Kruskal-Wallis test, Conover post hoc test and Jonckheere-Terpestra trend test were applied to assess the significance of differences, as parametric analyses were not possible. For all statistical tests, $P<0.05$ was considered statistically significant.

\section{Results}

A total of 250 eyes of 145 patients ( 77 females and 68 males) were included in this study: 117 eyes in the FLACS group and 133 in the CPS group. Subgroup A comprised eyes with NC 1-2 (35 FLACS-operated eyes and 42 CPSoperated eyes), subgroup B eyes with NC 3-4 (40 FLACSoperated eyes and 47 CPS-operated eyes), and subgroup C eyes with NC 5-6 (42 FLACS-operated eyes and 44 CPS-operated eyes). The number of cases was unequal in the main groups, since we chose cases from our private practice and the cost limited patients' choice between the procedures. Regarding mean age, there was no statistically significant difference between the FLACS and CPS groups $(62.5 \pm 8.1$ and $62.4 \pm 8.4$ years, respectively, $P=0.96)$ or among the subgroups: subgroup A; 60.2 \pm 11.6 in FLACS and $60.4 \pm 10.7$ years in CPS $(P=0.99)$, subgroup B; 60.3 \pm 4.6 in FLACS and 64.6 \pm 6.6 years in CPS $(P=0.54)$, and subgroup $C ; 62.8 \pm 6.8$ in FLACS and $62.2 \pm 7.3$ years in CPS $(P=0.77$; Table 1$)$.

The intraoperative parameters recorded in the two groups - EFX and US measures - are shown in Table 2. Nonsignificant differences in EFX split, EFX quadrant, average US\%, and total US time between the FLACS and CPS groups were found. EFX split was $9.96 \pm 8.8$ in the FLACS group and $10.28 \pm 8.35$ in the CPS group $(P=0.49)$. EFX quadrant was $17.33 \pm 14.22$ in the FLACS group and $19.96 \pm 15.1$ in the CPS group $(P=0.18)$. Average US was $5.9 \pm 3.3$ seconds in the FLACS group and $5.8 \pm 3.4$ in the CPS group $(P=0.45)$. Total US time was $72 \pm 45.8$ in the FLACS group and $66.5 \pm 45$ seconds in the CPS group $(P=0.44)$. As such, no statistically significant differences for these parameters were found (Table 2).

Table I Age differences among cases in FLACS and CPS groups and their subgroups

\begin{tabular}{|c|c|c|c|c|}
\hline & & $\mathbf{n}$ & Age (mean $\pm S D)$, years & $P$-value \\
\hline \multicolumn{2}{|l|}{ FLACS } & 117 & $62.5 \pm 8.1$ & \multirow{2}{*}{0.96} \\
\hline \multicolumn{2}{|l|}{ CPS } & 133 & $62.4 \pm 8.4$ & \\
\hline \multirow[t]{2}{*}{ Subgroup A } & FLACS & 35 & $60.2 \pm 11.6$ & \multirow[t]{2}{*}{0.99} \\
\hline & CPS & 42 & $60.4 \pm 10.7$ & \\
\hline \multirow[t]{2}{*}{ Subgroup B } & FLACS & 40 & $60.3 \pm 4.6$ & \multirow{2}{*}{0.54} \\
\hline & CPS & 47 & $64.4 \pm 6.6$ & \\
\hline \multirow[t]{2}{*}{ Subgroup $C$} & FLACS & 42 & $62.8 \pm 6.8$ & \multirow[t]{2}{*}{0.77} \\
\hline & CPS & 44 & $62.8 \pm 7.3$ & \\
\hline
\end{tabular}

Abbreviations: FLACS, femtosecond laser-assisted cataract surgery; CPS, conventional phacoemulsification..

Table 2 Intraoperative parameters in FLACS and CPS groups

\begin{tabular}{|l|l|l|l|}
\hline \multirow{2}{*}{} & $\begin{array}{l}\text { FLACS } \\
(\mathbf{n}=117)\end{array}$ & $\begin{array}{l}\text { CPS } \\
(\mathbf{n}=133)\end{array}$ & \multirow{2}{*}{-value } \\
\cline { 2 - 3 } & Mean \pm SD & Mean \pm SD & \\
\hline EFX split & $9.96 \pm 8.8$ & $10.28 \pm 8.35$ & 0.49 \\
EFX quadrant & $17.33 \pm 14.22$ & $19.96 \pm 15.1$ & 0.18 \\
Average US (\%) & $5.9 \pm 3.3$ & $5.8 \pm 3.4$ & 0.45 \\
Total US time & $72.0 \pm 45.8$ & $66.5 \pm 45.0$ & 0.44 \\
(seconds) & & & \\
\hline
\end{tabular}

Abbreviations: FLACS, femtosecond laser-assisted cataract surgery; CPS, conventional phacoemulsification; US, ultrasound. 
Table 3 Intraoperative parameters in FLACS and CPS subgroups

\begin{tabular}{|c|c|c|c|c|c|c|c|c|c|}
\hline & \multicolumn{3}{|l|}{ Subgroup A (NC I-2) } & \multicolumn{3}{|c|}{ Subgroup B (NC 3-4) } & \multicolumn{3}{|c|}{ Subgroup $C$ (NC 5-6) } \\
\hline & $\begin{array}{l}\text { FLACS }(n=35), \text { mean } \\
\pm \text { SD }\end{array}$ & $\begin{array}{l}\text { CPS }(n=42), \text { mean } \pm \\
\text { SD }\end{array}$ & $P$-value & $\begin{array}{l}\text { FLACS } \\
(n=40), \\
\text { mean } \pm \text { SD }\end{array}$ & $\begin{array}{l}\text { CPS } \\
(n=47), \\
\text { mean } \pm \\
\text { SD }\end{array}$ & $P$-value & $\begin{array}{l}\text { FLACS } \\
(n=42), \\
\text { mean } \pm \text { SD }\end{array}$ & $\begin{array}{l}\text { CPS } \\
(n=44), \\
\text { mean } \pm \\
\text { SD }\end{array}$ & $P$-value \\
\hline EFX split & $0.26 \pm 0.44$ & $0.45 \pm 0.5$ & 0.08 & $7.82 \pm 2.31$ & $\begin{array}{r}10.34 \\
\pm 2.83\end{array}$ & 0.0001 & $20.07 \pm 4.93$ & $19.59 \pm 4.7$ & 0.86 \\
\hline $\begin{array}{l}\text { EFX } \\
\text { quadrant }\end{array}$ & $2.11 \pm 1.64$ & $2.29 \pm 1.04$ & 0.49 & $14.3 \pm 5.92$ & $20 \pm 4.65$ & $<0.0001$ & $32.9 \pm 0.45$ & $36.8 \pm 8.98$ & 0.05 \\
\hline
\end{tabular}

Abbreviations: FLACS, femtosecond laser-assisted cataract surgery; CPS, conventional phacoemulsification; NC, nuclear cataract; US, ultrasound; EFX split, US energy required for nucleus splitting; EFX quadrant, US energy required for quadrant removal.

Intraoperative parameters recorded in the subgroups are shown in Table 3. In subgroup A, there were no significant differences between FLACS and CPS for EFX split or EFX quadrant ( $P=0.08$ and $P=0.49$ respectively). In subgroup $\mathrm{B}$, EFX split and EFX quadrant were significantly lower in the FLACS group than the CPS group $(P=0.0001$ and $P<0.0001$, respectively). Finally, for subgroup $\mathrm{C}$, a tendency toward lower values of EFX split and EFX quadrant was observed in the FLACS group, but difference in EFX split compared to the CPS group did not reach statistical significance $(P=0.86)$, while that for EFX quadrant was statistically significant $(P=0.05$, Table 3$)$.

\section{Discussion}

Use of the FL has been expanding recently in cataract surgery, specifically in creating corneal incisions, performing capsulotomies, and producing nuclear fragmentation. ${ }^{14,15}$ Many studies have reported the superiority of FLACS over CPS as regards US-energy consumption. ${ }^{15-17}$ A study in 2013 with the VICTUS platform (Bausch + Lomb, NJ, USA) found a significantly lower EPT in its FLACS group (5.2 \pm 5.7 seconds) than its CPS group ( $7.7 \pm 6.0$ seconds; $P=0.025) .{ }^{18}$ Similarly, Daya et al showed a significant reduction of $13.2 \%$ between their FLACS group $(8.58 \pm 4.66 \mathrm{sec}-$ onds) and CPS group ( $9.89 \pm 5.32$ seconds; $P=0.044) .{ }^{17}$

However, to our knowledge none of the available studies compared FLACS and CPS as regards US usage in the two specific phases of phacoemulsification surgery: nuclear splitting and quadrant removal. Therefore, in this study we compared the US-energy required for nucleus splitting (EFX split) and for quadrant removal (EFX quadrant) between FLACS and CPS in three subgroups with different nuclear densities.

We used lens-fragmentation patterns by FL, which has been demonstrated in previous studies as being the main factor contributing to the reduction in US energy in FLACS. ${ }^{15,19}$ In addition, we used $350 \mu \mathrm{m}$ grid softening, which has also been shown to lead to statistically significant lower EPT than other types of fragmentation grids. ${ }^{20}$ Moreover, we divided segmentation patterns into sextants. Based on our experience, this pattern helps in dividing the nucleus into small segments with subsequent easier manipulation and emulsification and less required US-energy.

In subgroup A (soft cataracts, NC 1-2), differences between FLACS and CPS concerning both EFX split and EFX quadrant were not significant $(P=0.08$ and 0.49 , respectively). This can be attributed to the already minimal or almost no US-energy required for both nuclear splitting and removal in CPS, owing to the fact that managing soft cataracts is more dependent on phacoemulsification fluidics than US-energy. This is most probably why FLACS was not superior to CPS in soft nuclear density.

As for subgroup B (medium density cataracts, NC 3-4), there was significantly lower US energy required in both splitting (EFX split) and quadrant (EFX quadrant) removal phases in favor of FLACS $(P=0.0001$ and $P<0.0001$, respectively). This was due to the efficiency of the FL in achieving complete division of the nucleus and effective softening of the nuclear quadrants, resulting in very minimal or no need for further US-energy for removal of nuclear parts.

Strikingly, in subgroup $\mathrm{C}$ (hard cataracts, NC 5$6)$, the difference in EFX split between FLACS and CPS was not significant $(P=0.86)$. This was probably due to the incomplete nucleus splitting we faced in FLACS-operated eyes. Although we increased the segmentation-repetition rate up to sixfold in this subgroup with the aim of increasing the frequency of FL pulses, we achieved neither complete segmentation nor nucleus splitting in any of those eyes. The FL did not affect the 


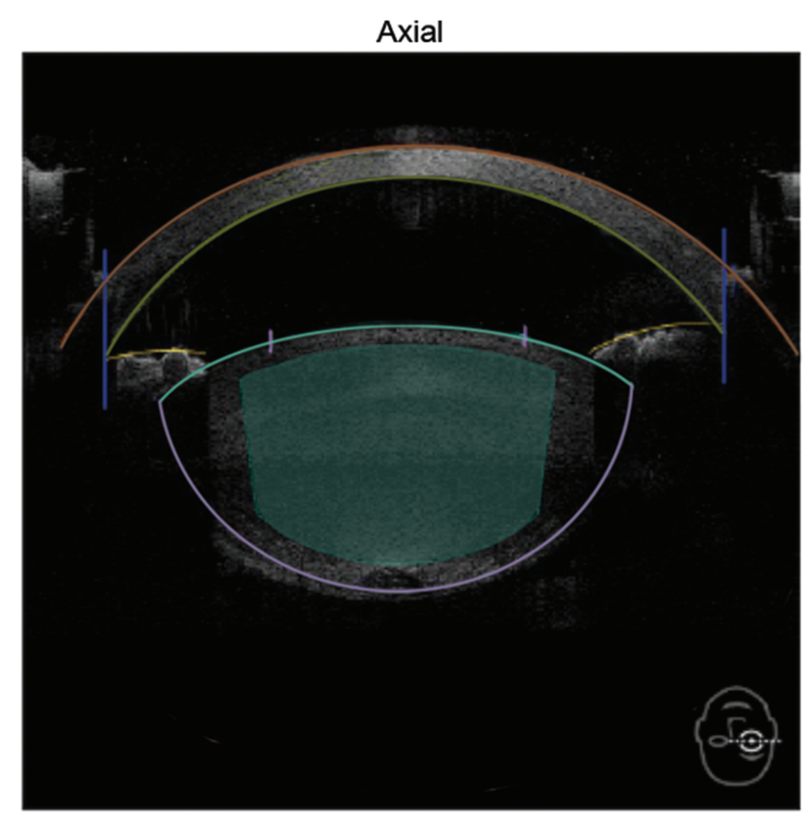

Figure 3 Anterior-segment axial scan by spectral-domain OCT of Catalys precision-laser system. Dense cataracts were noticed to extend posteriorly beyond the minimum laser-fragmentation offset of $500 \mu \mathrm{m}$ from the posterior capsule.

posterior parts of such thick and dense nuclei, as the Catalys laser-precision system does not allow extending of the laser beyond $500 \mu \mathrm{m}$ off the identified posterior capsule (Figure 3). This resulted in the need for subsequent US power to complete the step of nucleus cracking mechanically, with almost similar requirement of US energy as the CPS group (Figure 4).

It is important to say that we used $21 \mathrm{G}$ laminar-flow phacoemulsification needles in this study, as in all our routine cataract surgeries. This needle has an inner diameter of $0.5 \mathrm{~mm}$, and we assume that a 19 $G$ phacoemulsification needle with a larger inner diameter $(0.7 \mathrm{~mm})$ would help to aspirate chunks of prefragmented nuclei by vacuum with less US energy. A wide phacoemulsification needle might add a further reduction in US energy. However, we do not have a clear conclusion yet, and this matter is currently under investigation.

\section{Conclusion}

FLACS was highly beneficial in medium-density NCs, as it significantly reduced US-energy during both nucleus cracking and removal. Regarding hard cataracts, FLACS was only capable of reducing US energy during the quadrant-removal phase, in contrast to nucleus cracking, in which were inable to induce significant effects. As for soft cataracts, FLACS may be considered for benefits other than reduction in US energy.

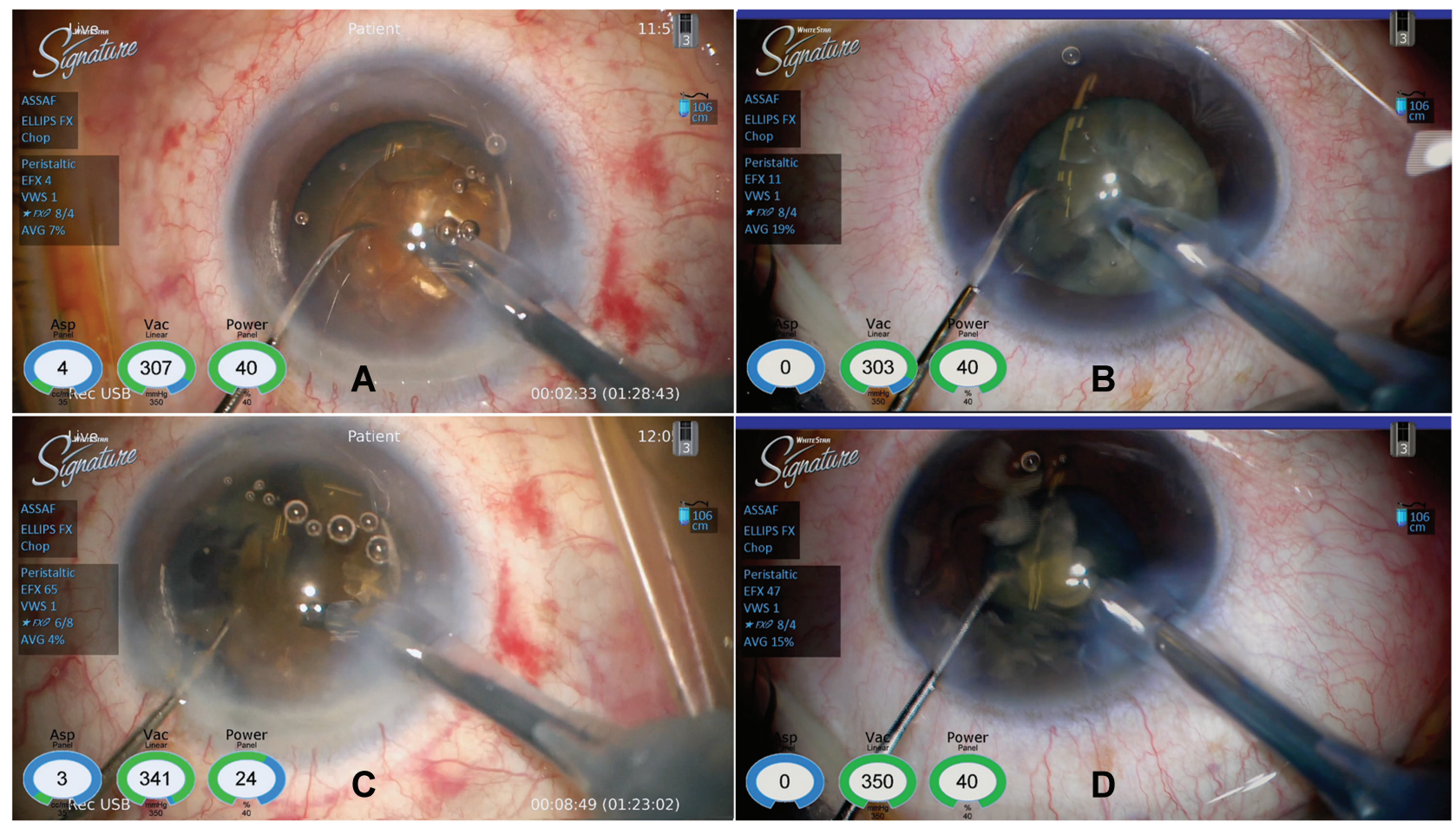

Figure 4 Dense (brunescent) cataract subgroup C. Nucleus-cracking phase. Similar US-energy levels were utilized in FLACS (A) and CPS (B). During quadrant removal, less US energy was utilized in the FLACS (C) than the CPS (D) subgroups. 


\section{Abbreviations}

CPS, conventional phacoemulsification surgery; EPT, effective phacoemulsification time; FL, femtosecond laser; FLACS, FLemtosecond-assisted cataract surgery; $\mathrm{G}$, gauge; IOL, intraocular lens;; $\mathrm{NC}$, nuclear cataract; PNS, Pentacam nucleus staging; US, ultrasound; EFX split, US energy required for nucleus cracking; EFX quadrant, US energy required for quadrant removal.

\section{Disclosure}

The authors report no conflicts of interest in this work.

\section{References}

1. Erie JC, Baratz KH, Hodge DO, Schleck CD, Burke JP. Incidence of cataract surgery from 1980 through 2004: 25-year population-based study. J Cataract Refract Surg. 2007;33(7):1273-1277. doi:10.1016/j. jers.2007.03.053

2. Bell CM, Hatch WV, Cernat G, Urbach DR. Surgeon volumes and selected patient outcomes in cataract surgery: a population-based analysis. Ophthalmology. 2007;114(3):405-410. doi:10.1016/j. ophtha.2006.08.036

3. Trikha S, Turnbull AM, Morris RJ, Anderson DF, Hossain P. The journey to femtosecond laser-assisted cataract surgery: new beginnings or a false dawn? Eye (Lond). 2013;27(4):461-473. doi:10.1038/ eye. 2012.293

4. Soong HK, Malta JB. Femtosecond lasers in ophthalmology. Am J Ophthalmol. 2009;147(2):189-197. doi:10.1016/j.ajo.2008.08.026

5. Nagy ZZ. New technology update: femtosecond laser in cataract surgery. Clin Ophthalmol. 2014;8:1157-1167. doi:10.2147/OPTH. S36040

6. Ang RET, Quinto MMS, Cruz EM, Rivera MCR, Martinez GHA. Comparison of clinical outcomes between femtosecond laser-assisted versus conventional phacoemulsification. Eye Vis. 2018;5(1):8. doi:10.1186/s40662-018-0102-5

7. He L, Sheehy K, Culbertson W. Femtosecond laser-assisted cataract surgery. Curr Opin Ophthalmol. 2011;22(1):43-52. doi:10.1097/ ICU.0b013e3283414f76

8. Chen X, Xiao W, Ye S, Chen W, Liu Y. Efficacy and safety of femtosecond laser-assisted cataract surgery versus conventional phacoemulsification for cataract: a meta-analysis of randomized controlled trials. Sci Rep. 2015;5(1):13123. doi:10.1038/srep13123

9. Kra'nitz K, Miha'ltz K, Sa'ndor GL, Takacs A, Knorz MC, Nagy ZZ. Intraocular lens tilt and decentration measured by scheimpflug camera following manual or femtosecond laser-created continuous circular capsulotomy. J Refract Surg. 2012;28(4):259-263. doi:10.3928/ 1081597X-20120309-01
10. Naranjo-Tackman R. How a femtosecond laser increases safety and precision in cataract surgery? Curr Opin Ophthalmol. 2011;23:53-57.

11. Berk TA, Schlenker MB, Campos-Mo"ller X, Pereira AM, Ahmed IIK. Visual and refractive outcomes in manual versus femtosecond laser-assisted cataract surgery: a single-center retrospective cohort analysis of 1838 eyes. Ophthalmology. 2018;125 (8):1172-1180. doi:10.1016/j.ophtha.2018.01.028

12. Nagy ZZ. Advanced technology IOLs in cataract surgery: pearls for successful femtosecond cataract surgery. Int Ophthalmol Clin. 2012;52(2):103-114. doi:10.1097/IIO.0b013e31824b452a

13. Conrad-Hengerer I, Al Sheikh M, Hengerer FH, Schultz T, Dick HB. Comparison of visual recovery and refractive stability between femtosecond laser-assisted cataract surgery and standard phacoemulsification: six-month follow-up. J Cataract Refract Surg. 2015;41 (7):1356-1364. doi:10.1016/j.jcrs.2014.10.044

14. Abell RG, Kerr NM, Vote BJ. Femtosecond laser-assisted cataract surgery compared with conventional cataract surgery. Clin Exp Ophthalmol. 2013;41(5):455-462. doi:10.1111/ceo.12025

15. Conrad-Hengerer I, Hengerer FH, Schultz T, Dick HB. Effect of femtosecond laser fragmentation on effective phacoemulsification time in cataract surgery. J Refract Surg. 2012;28(12):879-883. doi:10.3928/1081597X-20121116-02

16. Nagy Z, Takacs A, Filkorn T, Sarayba M. Initial clinical evaluation of an intraocular femtosecond laser in cataract surgery. J Refract Surg. 2009;25:1053-1060. doi:10.3928/1081597X-20091117-04

17. Daya SM, Nanavaty MA, Espinosa-Lagana MM. Transleticular hydrodissection, lens fragmentation, and influence on ultrasound power in femtosecond laser-assisted cataract surgery and refractive lens exchange. J Cataract Refract Surg. 2014;40(1):37-43. doi:10.1016/j.jcrs.2013.07.040

18. Reddy KP, Kandulla J, Auffarth GU. Effectiveness and safety of femtosecond laser-assisted lens fragmentation and anterior capsulotomy versus the manual technique in cataract surgery. $J$ Cataract Refract Surg. 2013;39(9):1297-1306. doi:10.1016/j.jcrs.2013.05.035

19. Kim DH, Wee WR, Lee JH, Kim MK. The comparison between torsional and conventional mode phacoemulsification in moderate and hard cataracts. Korean J Ophthalmol. 2010;24(6):336-340. doi:10.3341/kjo.2010.24.6.336

20. Conrad-Hengerer I, Hengerer FH, Schultz T, Dick HB. Effect of femtosecond laser fragmentation of the nucleus with different softening grid sizes on effective phaco time in cataract surgery. J Cataract Refract Surg. 2012;38(11):1888-1894. doi:10.1016/j. jcrs.2012.07.023
Clinical Ophthalmology

\section{Publish your work in this journal}

Clinical Ophthalmology is an international, peer-reviewed journal covering all subspecialties within ophthalmology. Key topics include: Optometry; Visual science; Pharmacology and drug therapy in eye diseases; Basic Sciences; Primary and Secondary eye care; Patient Safety and Quality of Care Improvements. This journal is indexed on PubMed

Submit your manuscript here: https://www.dovepress.com/clinical-ophthalmology-journal

\section{Dovepress}

Central and CAS, and is the official journal of The Society of Clinical Ophthalmology (SCO). The manuscript management system is completely online and includes a very quick and fair peer-review system, which is all easy to use. Visit http://www.dovepress.com/ testimonials.php to read real quotes from published authors. 